\title{
Elite reviewers 2011
}

\author{
Richard C. Becker
}

Published online: 24 December 2011

(C) Springer Science+Business Media, LLC 2011

As the founding Editor-in-Chief, and on behalf of our Editorial Board members and Associate Editors, I would like to extend a special thanks to our elite reviewers for their timely, thoughtful and exemplary approach to a time honored process that has its roots in the annals of science and medicine; a tenet that serves both as a bedrock and fabric for the scholarly exchange of ideas and the dissemination of knowledge among scientists-past, present and future.

The promotion of peer review and the practice of scientists independently evaluating each other's work dates back to the Royal Society and its establishment, in 1665, of the first peer reviewed scientific journal-Philosophical Transactions [1]. Despite having its critics, peer review in its most fundamental and purest form, is the best means available to subject scholarly work to the scrutiny of experts in the field who are qualified, experienced and fully expected by their peers and by the scientific community at large to perform an impartial and unbiased review. Indeed, an elite reviewer recognizes their important role in securing the integrity of published work and embraces the responsibility, not merely by providing a "yes or no" judgment of publishability, but through a dedicated, rigorous and constructive assessment of a study or experiment's purpose, methods, results, interpretation and translatability in a selected subject of inquiry.

As the Editor-in-Chief, I have from the Journal's inception in 1994, recognized the importance of selecting reviewers to serve as referees of submitted manuscriptsbeing ever mindful of potential conflicts, bias and other

R. C. Becker $(\bowtie)$

Duke University Medical Center,

Durham, NC, USA

e-mail: richard.becker@duke.edu barriers to fulfilling our mission to provide a respected resource for clinicians and scientists active in the fields of cardiology, hematology, pharmacology and vascular biology. In this issue of the Journal, we not only acknowledge our elite reviewers, but pledge an oath to the process of peer review in its most honest, open, independent and ethical form.

\section{Journal of Thrombosis and Thrombolysis elite reviewers 2012}

Jens D. Lohrmann

Felicita Andreotti

Paul Y. Kim

Michael Wagner

Frederick A. Spencer

Ian J. Welsby

Peter Whittaker

Jack Ansell

Jeffrey Washam

Christoph Bode

Alessandro Squizzato

Norbert Gerdes

Jeffrey Weitz

Susan Smyth

\section{Reference}

1. Becker KC (2010) The world through a lens: a history of the royal society of London, and the discovery of the red blood cell. J Thromb Thrombolysis 30:496-499 